\title{
Efecto de períodos de ayuno en el crecimiento de la zoea I de Petrolisthes violaceus (Guérin, 1831) (Decapoda: Anomura: Porcellanidae)
}

\author{
Luis M. Pardo ${ }^{1}$, David Véliz ${ }^{1}$, Ingo S. Wehrtmann ${ }^{2}$ y Klaus Anger ${ }^{3}$ \\ ${ }^{1}$ Departamento de Biología Marina, Universidad Católica del Norte \\ Casilla 117, Coquimbo, Chile \\ ${ }^{2}$ Instituto de Zoología, Universidad Austral de Chile, Casilla 567, Valdivia, Chile \\ ${ }^{3}$ Biologische Anstalt Helgoland, Postfash 180, 27483, Helgoland, Alemania
}

\begin{abstract}
RESUMEN. El presente estudio describe las consecuencias del ayuno en el crecimiento (peso húmedo y seco) y la supervivencia de la primera zoea de Petrolisthes violaceus, una especie común en el litoral chileno. Se realizaron cinco grupos experimentales ( 1 hasta 5 días de alimentación después de la eclosión, respectivamente) más un tratamiento de ayuno y un control con alimentación diaria. El alimento (Artemia franciscana) consistió en 15 nauplius $/ \mathrm{ml}$. La temperatura se mantuvo a $19^{\circ} \mathrm{C}$ y la salinidad a $34^{\circ} \%$. El mayor crecimiento en biomasa se logró en larvas con alimentación diaria, con una ganancia neta de $94,7 \%$ en peso seco con respecto a las recién eclosionadas. En los otros experimentos (2-5 días de alimentación inicial) se observó un leve incremento de la biomasa. Las larvas con un día de alimento y las mantenidas en ayuno tuvieron pérdidas de biomasa; estas últimas disminuyeron sostenidamente su biomasa hasta perder el 18,9\% de su peso seco inicial. El Punto de Saturación de Reserva (PRS) se obtuvo aproximadamente a los 4 días de desarrollo. La zoea I de $P$. violaceus tiene una evidente vulnerabilidad al ayuno, ya que no es capaz de completar su ciclo de muda en ausencia o con períodos cortos (1-3 días) de alimentación. Además, las larvas sólo mudaron cuando aumentaron más de la mitad de su peso corporal inicial (55,4\%). Los resultados de los tratamientos 1-5 permiten postular que las larvas poseen una alta capacidad de asimilación ya que tienen pérdida de biomasa sólo dos días después de someterlas a ayuno. Esta capacidad permitiría compensar la pérdida de biomasa causada por el ayuno.
\end{abstract}

Palabras claves: ayuno, zoea, crecimiento, PRS, Porcellanidae.

\section{Effects of starvation periods on growth of zoea I in Petrolisthes violaceus (Decapoda: Anomura: Porcellanidae)}

\begin{abstract}
The present study describes consequences of starvation upon growth (wet and dry weight) and survival of zoea I - larvae of Petrolisthes violaceus, a common species of the Chilean intertidal belt. The experimental design included five groups (1-5 days after hatching without food, respectively) as well as one starvation treatment and one control (larvae were fed daily). Food (Artemia franciscana) consisted in 15 nauplii per ml. Temperature was kept at $19^{\circ} \mathrm{C}$ and salinity at $34 / 00$. The larvae which were fed daily achieved the highest biomass increase, gaining $94.7 \%$ of its initial dry weight. In other experiments (2-5 days of initial feeding) a slight biomass increase was observed. Larvae fed only one day and those under starvation conditions lost biomass: starved larvae a continuos biomass decrease up to $18.9 \%$ of its initial dry weight. The "Point of Reserve Saturation" was at 4 days. The first zoeal stage of $P$. violaceus demostrated a low starvation resistance, since zoea I was not capable to complete the molt cycle in the absence or with brief periods (1-3 days) of food. Furthermore, larvae molted only when they inceased their initial body weight by more then half (55.4\%). Our results suggest that larvae of $P$. violaceus possess a high capacity of assimilation since they start loosing biomass not before two days of starvation.This capacity may permit to compensate the biomass loss caused by food deprivation.
\end{abstract}

Key words: starvation, zoea, growth, PRS, Porcellanidae. 


\section{INTRODUCCION}

Un gran número de invertebrados marinos bentónicos poseen períodos larvales planctónicos, ya sea, lecitotróficos o planctotróficos. Este período es de suma importancia, ya que condiciona el reclutamiento a la población de individuos jóvenes y la dispersión de la especie. Para larvas plantotróficas, la temperatura, predación y disponibilidad de alimento son los principales factores que afectan la supervivencia larval (Thorson, 1950).

Existen numerosos estudios sobre el crecimiento, duración de los estadíos y mortalidad larval en crustáceos decápodos sometidos a diferentes temperaturas y/o salinidades, distintos tipos de dieta y cantidad de alimento. De estos se puede extraer que una disminución en la cantidad de alimento provoca una baja en el crecimiento e incrementa el riesgo de mortalidad por ayuno o predación, al aumentar el período de duración larval (Sulkin, 1975; Anger et al., 1981; Dawirs, 1982, 1983; McConaugha, 1985; Harms y Seeger, 1989).

Anger (1987) determina, para algunas especies de decápodos, puntos críticos del ciclo de la muda en un estadío larval. Estos puntos pueden ser asociados a) períodos iniciales de alimentación en que las larvas alcanzan a almacenar un mínimo de sustancias esenciales para lograr con éxito el siguiente estadio, aunque después de este período se les prive del alimento (Punto de saturación de reserva: PRS) o b) períodos iniciales de ayuno, en que las larvas sufren daños irreversibles y no alcanzan el siguiente estadío a pesar de ser alimentadas posteriormente (Punto de no retorno: PNR). Estos puntos críticos pueden ser de gran valor ecológico, como una adaptación fisiológica de las larvas de decápodos a las condiciones de impredicibilidad del ambiente planctónico, larvas con un bajo valor de PRS y un alto PNR serían capaces de resistir de una mejor manera las condiciones de escasez de alimento.

Petrolisthes violaceus (Guérin, 1831) es un crustáceo intermareal distribuido desde Bahía de Ancón, Perú hasta Taitao, Aisén, Chile (Retamal, 1981). Su desarrollo larval está descrito por Wehrtmann et al. (1997) y consta de dos zoeas y una megalopa.

El presente trabajo tiene como objetivo establecer el crecimiento de la zoea I de $P$. violaceus y estimar la biomasa y tiempo mínimo a adquirir para lograr el siguiente estadío, discutiendo su posible implicancia ecológica para la vida planctónica.

\section{MATERIALES Y METODOS}

Hembras ovígeras de Petrolisthes violaceus se colectaron desde el intermareal rocoso de la concesión marítima de la Universidad Católica del Norte, ubicada en Bahía La Herradura, Coquimbo (2958'S; 71²2'W), en marzo de 1995. Se eligieron sólo las hembras que poseían huevos en estadío III avanzado (sensu Lardies y Wehrtmann, 1996), las que fueron posteriormente transportadas a los laboratorios de la Universidad Católica del Norte y puestas en acuarios de 20 litros con agua de mar filtrada a $1 \mu$ y aireación constante.

Se obtuvo una liberación simultánea de larvas de cuatro hembras de $P$. violaceus (longitud cefalotoráxica de 19,5 $\pm 0,5 \mathrm{~mm}$ ). Estas zoeas fueron mezcladas y colocadas en frascos con $500 \mathrm{ml}$ de agua de mar filtrada a $1 \mu$ y esterilizada con luz ultravioleta (50 larvas por frasco).

Se mantuvieron 5 tratamientos, que van de 1 hasta 5 días de alimentación después de la eclosión, además un tratamiento de ayuno y un control con alimentación diaria (Fig. 1). El total de larvas utilizadas fue de 2150. El alimento consistió en 15 nauplios de Artemia franciscana (San Francisco Bay Brand) por ml de agua (Anger, datos no publicados). La temperatura durante la experimentación fue de $19^{\circ} \mathrm{C}$ y la salinidad $34^{\circ} \%$. Para determinar el crecimiento en biomasa, se extrajeron diariamente un total de 30 larvas por cada grupo experimental, incluyendo el tratamiento de ayuno y el control alimentado diariamente, a los cuales se les determinó el peso húmedo y peso seco en una balanza Precisa 100A-300M con una precisión de $0,1 \mathrm{mg}$. El peso húmedo se obtuvo inmediatamente después de extraer el excedente de agua de las larvas con un papel filtro y el peso seco después de estar 24 horas en la estufa a $70^{\circ} \mathrm{C}$.

A partir de la ecuación de regresión del porcentaje de las larvas mudadas a zoea II en los distintos tratamientos, se interpoló el tratamiento (cantidad de días con alimento) que correspondió al 50\% de sobrevivencia (PRS50). Este valor corresponde al tiempo (en días) en el cual se obtuvo el $50 \%$ de larvas mudadas, tomando el $100 \%$ como el total de las larvas mudadas del control. Además, se realizaron observaciones a microscopía óptica de luz de las larvas recién muertas, para comprobar su estadío dentro del ciclo de muda.

Finalmente, se calcularon los porcentajes de supervivencia al estadío de zoea II en los distintos tra- 


\begin{tabular}{|c|c|c|c|c|c|c|c|}
\hline & & & \multicolumn{5}{|c|}{ Lias } \\
\hline TRATAMIENTOS & 1 & 2 & 3 & 4 & 5 & 6 & 7 \\
\hline $\mathrm{A}$ & & & & & & & \\
\hline $\mathrm{T} 1$ & & & & & & & \\
\hline $\mathrm{T} 2$ & & & & & & & \\
\hline $\mathrm{T} 3$ & & & & & & & \\
\hline $\mathrm{T} 4$ & & & & & & & \\
\hline $\mathrm{T} 5$ & & & & & & & \\
\hline $\mathrm{C}$ & & & & & & & \\
\hline
\end{tabular}

Figura 1. Esquema del tratamiento experimental. Los cuadros blancos muestran los días en que las larvas se sometieron a ayuno y los de color gris, los días en que se les otorgó alimento.

Figure 1. Experimental treatment design. White squares show the days in which the larvae were subject to fast and the gray squares the days in which were fed.

tamientos, los que se compararon estadísticamente a través de una prueba de chi-cuadrado en una tabla de contingencia de 2x2 (Sokal y Rholf, 1984).

\section{RESULTADOS}

La variación del porcentaje de agua corporal (estimado a través de la diferencia entre el peso húmedo y el peso seco) se representa en la Fig. 2. Para todos los tratamientos, los valores de peso húmedo y cantidad de agua corporal, descienden a través de los días, teniendo posteriormente un incremento el día 7, excepto en el tratamiento de ayuno, donde el peso húmedo desciende gradualmente hasta $480 \mathrm{~g} / \mathrm{ind}$. y el porcentaje de agua corporal, se mantiene constante alrededor del $82 \%$.

Las ganancias netas de biomasa, medidas en peso seco, se muestran en la Fig. 3. Se observa que sólo las larvas del tratamiento de ayuno (A) y un día de alimento (T1) muestran una pérdida de peso, con respecto al inicial. El resto de los tratamientos muestran un aumento en el peso larval. Considerando que el día 7 post-eclosión se producen las primeras mudas en el control, el PRS50 de la zoea I se alcanza aproximadamente al día 4, con un peso seco de $137 \mathrm{mg} /$ ind.

En los tratamientos en los cuales se logra el paso a zoea II (T4 y T5), a pesar de tener un período de ayuno, la ganancia neta de peso seco de la zoea I es de $65,5 \%$ y $55,4 \%$, respectivamente, notoriamente inferior a la ganancia de las larvas con alimento diario $(94,74 \%)$, donde se duplica el valor inicial. La biomasa adquirida por los tratamientos 2 y 3 fue insuficiente para lograr el paso al siguiente estadío. Las larvas que permanecieron en ayuno o con sólo un día de alimentación presentaron una pérdida de biomasa del $18,9 \%$ y $2,1 \%$ del peso inicial respectivamente (Fig. 4a).
La supervivencia de las larvas medida como porcentaje de individuos que llegaron a zoea II desde la eclosión (Fig. 4b) muestra un valor significativamente inferior para las larvas con cuatro días de alimento (T4) en comparación con el T5 $(\mathrm{X} 2=4,86 ; \mathrm{p}<0,05)$. El tratamiento 5 y Control no muestran diferencias estadísticas significativas $(\mathrm{X} 2=0,45 ; \mathrm{p}>0,05)$.

La supervivencia máxima para las larvas en ayuno fue de 5 días, 8 para el T1 y de 10 días para las larvas de T2 y T3. La media ponderada de duración del primer estadío de zoea para T4 fue de 7,75 días, para T5 de 8,11 y para el Control 9,14 días.

En la observación microscópica de las larvas muertas de las tratamientos de ayuno, T1, T2, T3 y $\mathrm{T} 4$, se puede separar claramente la etapa del ciclo en que se detuvo la muda (sensu Drach, 1939). En las larvas en ayuno y del T1 se observaron las delimitaciones cuticulares bien definidas, pero no se observa una separación de la cutícula con la epidermis (Estadío de Intermuda: C). En cambio, en las larvas alimentadas los primeros 2 y 3 días, se observa claramente la apólisis (separación de la epidermis con la vieja cutícula) y la nueva cutícula completamente formada; además se evidencian levemente algunas invaginaciones epidermales (Estadío de Premuda Tardía: D2-4). Las larvas con alimento continuo muestran un desarrollo morfológico normal del ciclo de muda.

\section{DISCUSION}

Generalmente, el punto de saturación de reserva de los primeros estadíos de decápodos se encuentra entre el tercio o la mitad de la duración del período larval (Anger, 1987). Esto indica, que las zoeas I de $P$. violaceus poseen una evidente vulnerabilidad al ayuno, ya que las larvas necesitan ser alimentadas al menos el $57 \%$ del período de la primera zoea. 

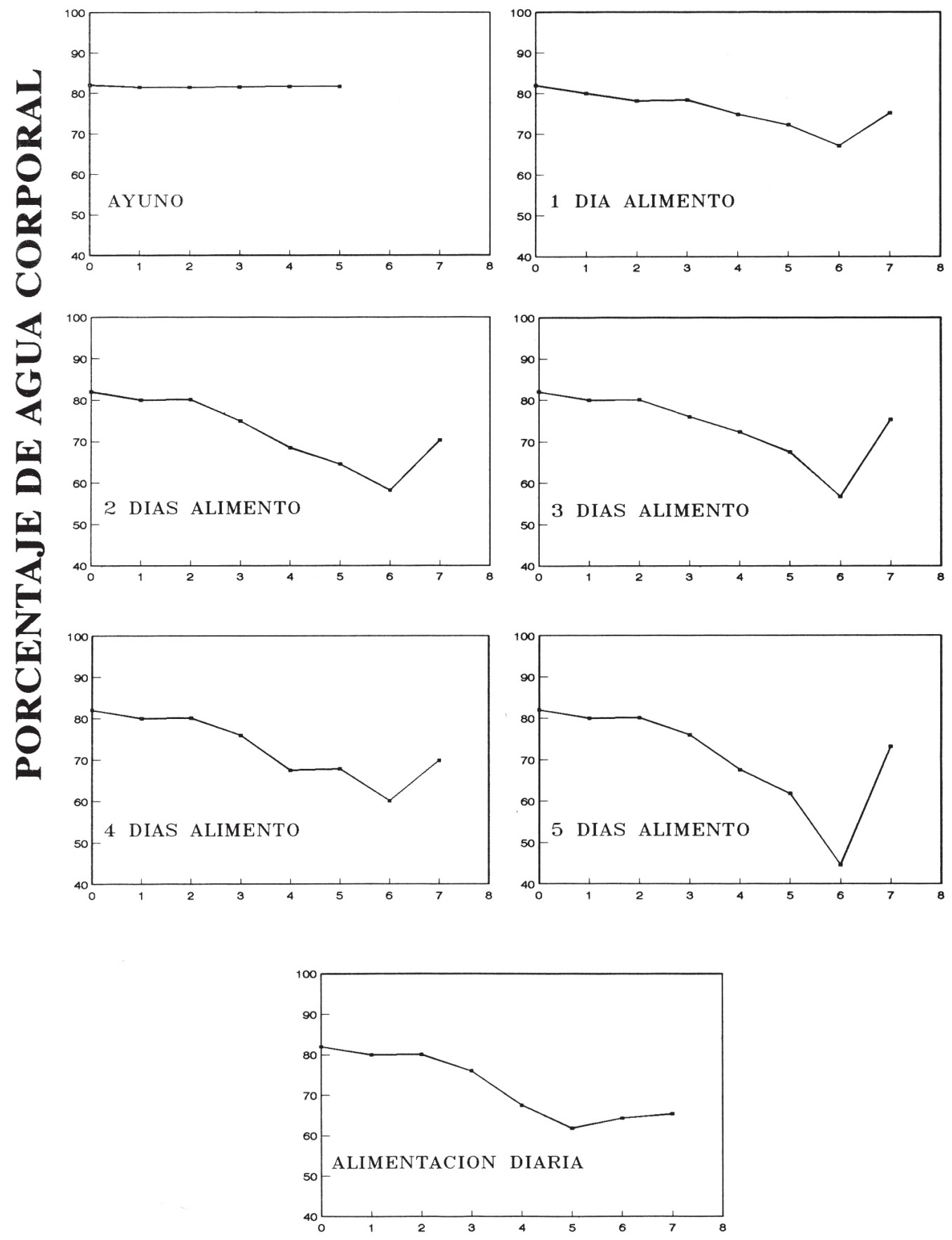

DIAS

Figura 2. Variación diaria del porcentaje de agua corporal de las larvas de Petrolisthes violaceus para todos los tratamientos y el control (alimentación).

Figure 2. Daily variation of the body water percentage of Petrolisthes violaceus larvae in all treatments and the control (daily feeded). 


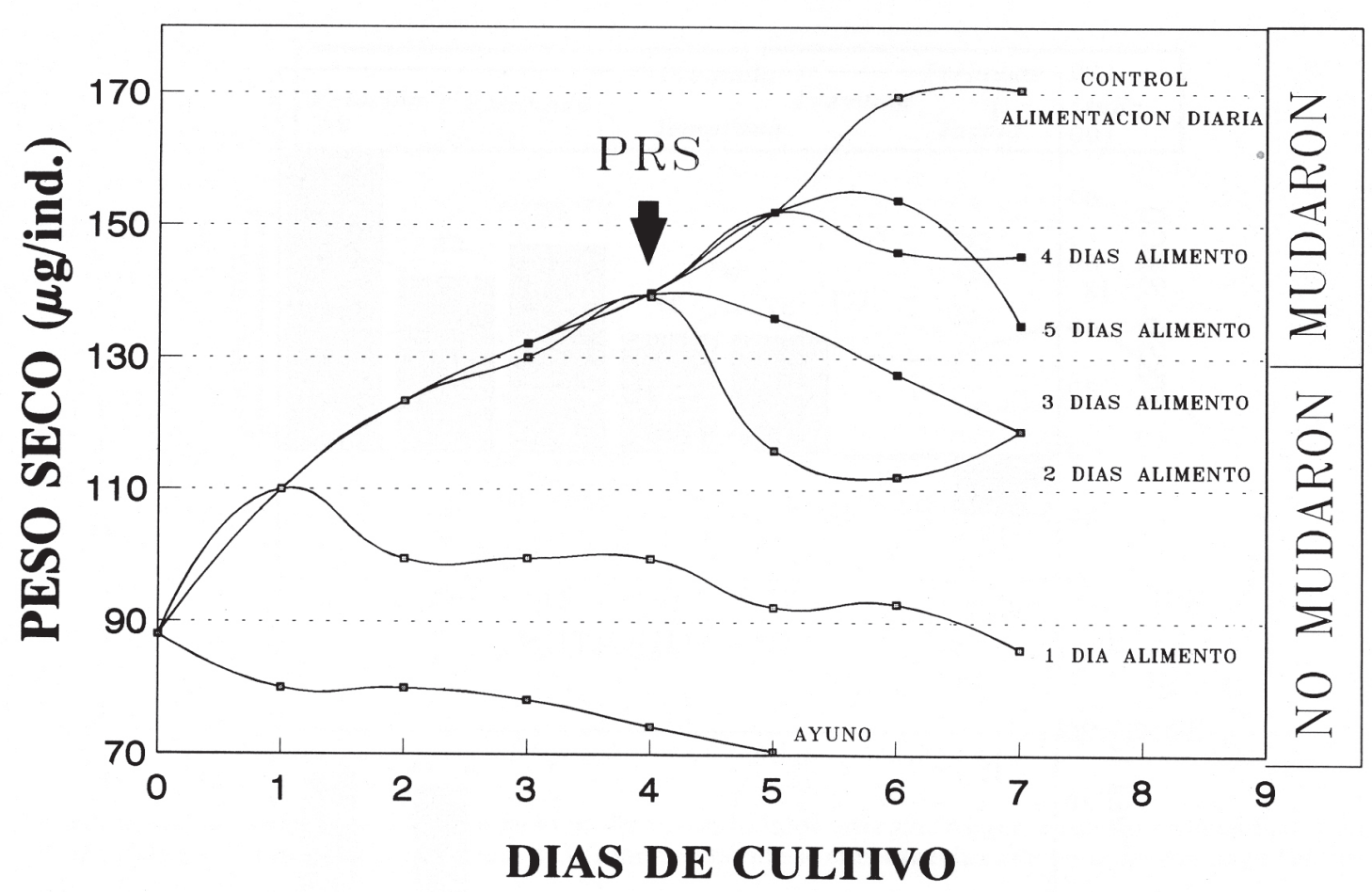

Figura 3. Variación diaria del peso seco de las larvas de Petrolisthes violaceus para cada uno de los tratamientos, expresado en mg/ind. La gráfica además muestra el PRS (obtenido a los cuatro días de experimentación) y los tratamientos en los cuales se obtuvo muda (tratamientos 4, 5 y control).

Figure 3. Daily dry-weight variation of Petrolisthes violaceus larvae for each treatment, expressed in mg/ind. The grap also shows the PRS (obtained after the four days of experimentation) and the treatments in which molting was obtained (treatments 4,5 and control).

No se observó un aumento en el peso seco el primer día de desarrollo, en las larvas mantenidas en ayuno, como ocurre con otras especies (Anger, 1986; Dawirs, 1987). En este período inicial se consumirían las reservas vitelogenéticas para la etapa de hipertrofia celular, principalmente lípidos, los cuales son los sustratos energéticos para resistir los períodos sin alimentación (Anger y Spindler, 1987). Sin embargo, al estar sólo un día en contacto con el alimento, las larvas aumentan su biomasa y son capaces de prolongar su supervivencia.

El notorio aumento del porcentaje de agua corporal en el día siete (Fig. 2) refleja una evidente ganancia de agua en las larvas de los diferentes tratamientos, excepto aquellas mantenidas en ayuno. Esto puede significar que las larvas con muy poco alimento son capaces de llegar a fases de premuda tardía, en donde los espacios entre la vieja cutícula y la cutícula en formación son ocupadas con agua (Anger, 1983). Lo mismo ocurre en las zoeas mantenidas con alimento diario; sin embargo, su por- centaje de agua corporal no se eleva en el último día del ciclo de muda, debido a que es compensado con un crecimiento en biomasa.

Dos hechos demuestran la capacidad de las larvas con poco alimento inicial de pasar el umbral Do de premuda temprana (Anger, 1987), en donde se produce la apólisis o separación de la epidermis y la vieja cutícula. Primero, el largo período (10 días) de supervivencia de las larvas alimentadas más de un día y segundo, la comprobación microscópica del estadío de premuda tardía (D2-D4) en las larvas alimentadas 2 a 3 días (T2 y T3). En este estadío de premuda tardía ocurriría la formación de la nueva cutícula (Anger, 1983) con una consecuente demanda energética. Este sería un segundo punto crítico en el ciclo de la muda de larvas de decápodos (Anger, 1987). Con los antecedentes del presente trabajo se confecciona un modelo teórico para el ciclo de la muda de la zoea I de $P$. violaceus (Fig. 5). 

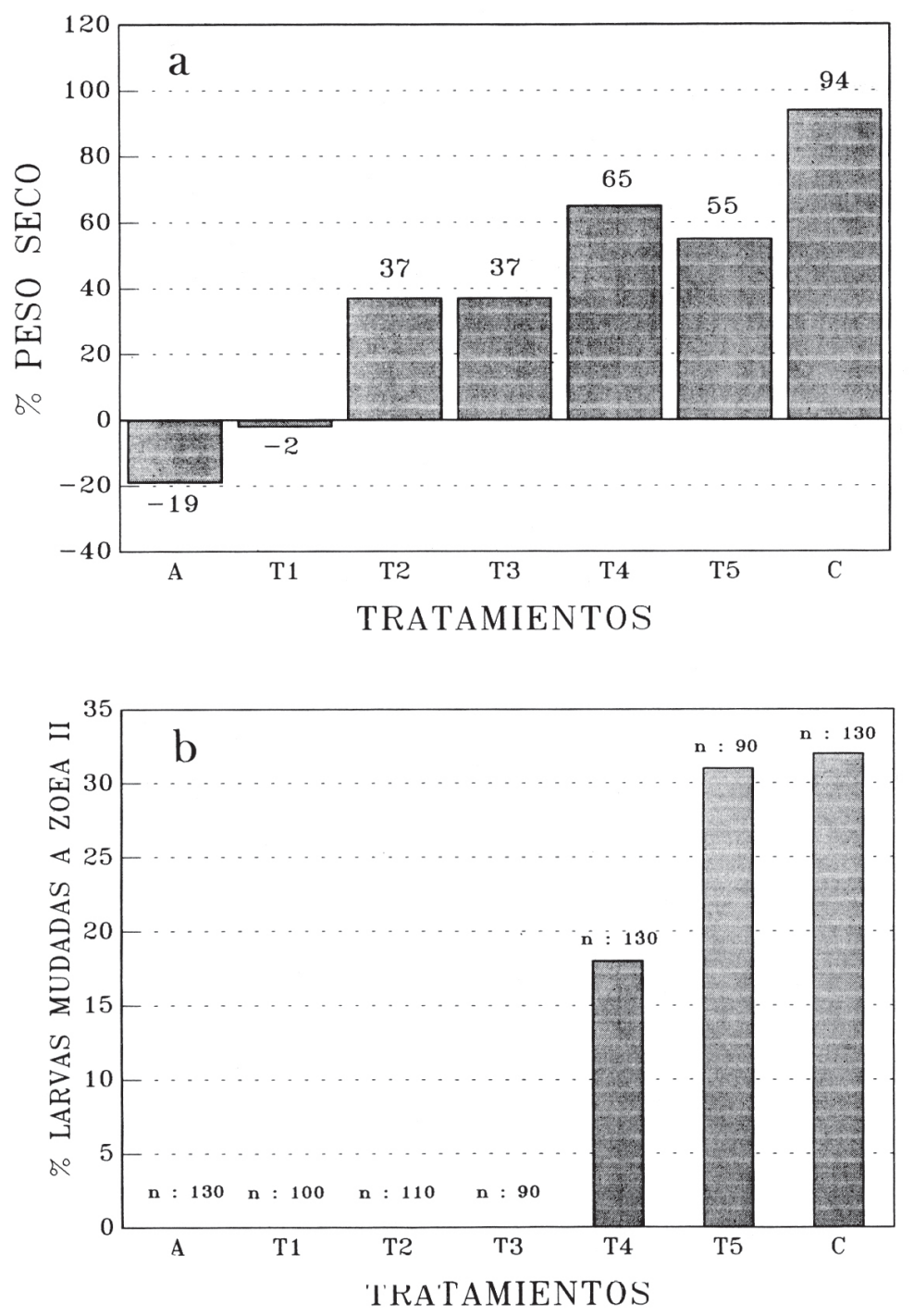

Figura 4. Larvas de Petrolisthes violaceus. 4a: Ganancias y pérdidas de biomasa para los diferentes tratamientos en el séptimo día de experimentación, en relación al peso seco inicial. 4b: Porcentaje de las larvas que mudaron a zoea II: No existen diferencias significativas entre el tratamiento 5 y control $(X 2=0,45 ; p>0,05)$. El tratamiento 4 difiere significativamente del tratamiento $5(X 2=4,86 ; p<0,05)$.

Figure 4. Petrolisthes violaceus larvae. 4a: Biomass gain and looses for different treatments in the seventh day of experimentation, relative to the initial dry-weigth. 4b: Percentage of larvae molting to zoea II. No significant differences between treatments 5 and control were observed $(X 2=0,45 ; p>0,05)$. Treatment 4 differs significantly from treatment $5(\mathrm{X} 2=4,86 ; \mathrm{p}<0,05)$.

Lo anteriormente dicho, más el hecho de que las zoeas disminuyen su biomasa sólo después de dos días de que se les privó del alimento (Fig. 3), supone una alta capacidad de asimilación, lo cual compensaría su baja resistencia al ayuno.

La biomasa mínima para mudar al siguiente estadío, corresponde a un 55\% más del peso de las larvas recién eclosionadas. Esto se logra después de
4 días de alimentación, pero la supervivencia de la zoea II es significativamente inferior que las larvas mejor alimentadas. Al respecto, sólo existen datos para Carcinus maeneas, en donde las larvas necesitan un aumento del $20 \%$ de su peso inicial para pasar al estadio II (Dawirs, 1983) y Hyas araneus donde la zoea I duplica su peso seco desde la eclosión 


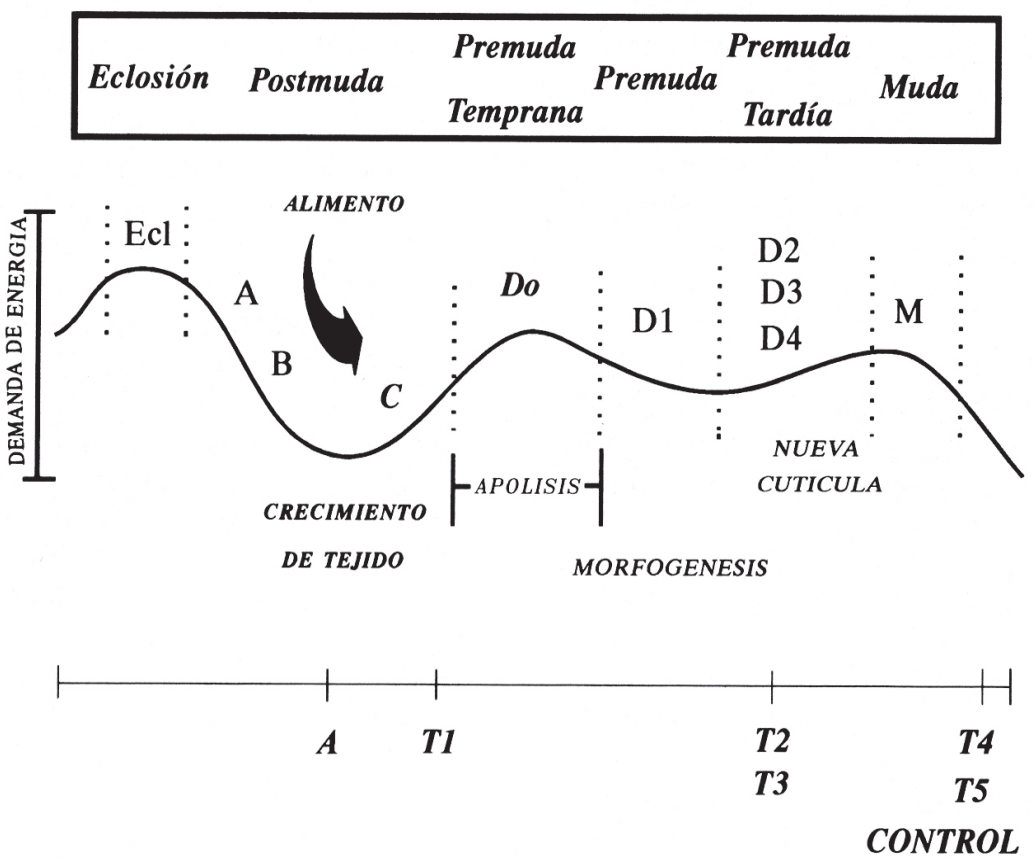

Figura 5. Modelo teórico para el ciclo de la muda y demanda de energía (Anger, datos no publicados). La línea inferior indica el estadío dentro del ciclo de la muda en donde se encontraría cada tratamiento para Petrolisthes violaceus.

Figure 5. Theoretical model for the molting cycle and energy demand (Anger, unpublished data). The lower line indicates the stage within the molting cycle in which each treatment for Petrolisthes violaceus would be.

hasta alcanzar el umbral Do (Anger y Spindler, 1987). Esta diferencia podría tener relación con el tipo de desarrollo de los tres decápodos, $P$. violaceus y $H$. araneus sólo tienen tres estadíos larvales (dos zoeas y una megalopa), en cambio $C$. maeneas posee cinco (cuatro zoeas y una megalopa). Este hecho podría indicar una mayor capacidad de asimilación (expresado en el porcentaje de biomasa adquirida) de las larvas de especies con un menor número de estadíos en su desarrollo. Futuros trabajos en crecimiento larval de otros grupos de crustáceos decápodos podrían apoyar esta idea.

\section{AGRADECIMIENTOS}

Queremos agradecer el apoyo ofrecido por la Universidad Católica del Norte (Departamento de Biología Marina) en la realización de este trabajo, en especial la cooperación de M. Sc. Chita Guisado. El presente estudio fue realizado durante el desarrollo de un curso de postgrado realizado en Coquimbo, el cual fue financiado por "Banco de Crédito e Inversiones", INFODEMA y EQUILAB
Ltda. Se agradece la revisión del escrito por parte de dos correctores anónimos. K. Anger agradece al "Servicio Alemán de Intercambio Académico" (DAAD), a la Universidad Austral de Chile, Valvidia, y a la Universidad Católica del Norte, por el subsidio económico brindado en su estadia y realización del curso.

\section{REFERENCIAS}

Anger, K. 1983. Moult cycle and morphogenesis in Hyas araneus larvae (Decapoda: Majidae), reared in the laboratory. Helgölander Meeresunters, 36: 285-302.

Anger, K. 1986. Changes of respiration and biomass of spider crab (Hyas araneus) larvae during starvation. Mar. Biol., 90: 261-269.

Anger, K. 1987. The Do threshold: a critical point in the larval development of decapod crustacans. J. Exp. Mar. Biol. Ecol., 108: 15-30. 
Anger, K. y K.D. Spindler. 1987. Energetics, moult cycle and ecdysteroid titers in spider crab (Hyas araneus) larvae. Starved after the Do threshold. Mar. Biol., 94: 367-375.

Anger, K., R. Dawirs, V. Anger y J. Costlow. 1981. Effects of early starvation periods on zoeal development of brachyuran crabs. Biol. Bull., 161: 199-212.

Dawirs, R. 1982. Methodological aspects of rearing decapod larvae Pagurus bernhardus (Paguridae) and Carcinus maeneas (Portunidae). Helgölander Meeresunters., 35: 434-464.

Dawirs, R. 1983. Respiration, energy balance and development during growth and starvation of Carcinus maeneas L. larvae (Decapoda: Portunidae). J. Exp. Mar. Biol. Ecol., 69: 105128.

Dawirs, R. 1987. Influence of limited starvation periods on growth and elemental composition $(\mathrm{C}, \mathrm{N}, \mathrm{H})$ of Carcinus maeneas (Decapoda: Portunidae) larvae reared in the laboratory. Mar. Biol., 91: 543-549.

Drach, P. 1939. Mue et cycle d'intermue chez les Crustacés décapodes. Ann. Inst. Océangr. (Monaco), 19: 103-391.

Harms, J. y B. Seeger. 1989. Larval development and survival in seven decapods species (Crustacea) in relation to laboratory diet. J. Exp. Mar. Biol. Ecol., 133: 129-139.

Recibido el 07 de mayo de 1996.

Aceptado el 23 de diciembre de 1996.
Lardies, M.A. y I.S. Wehrtmann. 1996. Aspects of the reproductive biology of Petrolisthes laevigatus (Guérin, 1835) (Decapoda: Anomura: Porcellanidae). I. Reproductive output and chemical composition of eggs during embryonic development. Arc. Fish. Mar. Res., 43(2): 121135.

McConaugha, J. 1985. Nutrion and larval growth. In: A.M. Wenner y A.A. Balkema (ed.), Larval growth. Rotterdam, pp. 127-154.

Retamal, M.A. 1981. Catálogo ilustrado de los Crustáceos Decápodos de Chile. Gayana, (zool.), 44: 1-110.

Sokal, R.R. y F.J. Rholf. 1981. Biometry. Second Edition. Freeman Press, San Francisco 859 pp.

Sulkin, S. 1975. The significance of diet in the growth and development of larval of the blue crab Callinectes sapidus, under laboratory conditions. J. Exp. Mar. Biol. Ecol., 20: 119135.

Thorson, G. 1950. Reproductive and larval ecology of marine bottom invertebrates. Biol. Rev., 25: $1-45$.

Wehrtmann, I.S., L. Albornoz, L.M. Pardo y D. Véliz. The larval development of Petrolisthes violaceus (Guérin, 1831) (Decapoda: Anomura: Porcellanidae) from Chilean waters, cultivated under laboratory conditions. Crustaceana, 70(5): 562-583. 